\title{
An Amperometric Immunosensor Based on a Conducting Immunocomposite Electrode for the Determination of Schistosoma japonicum Antigen
}

\author{
Ya-Min Zhou, Guo-Dong LiU, Zhao-Yang Wu, Guo-Li Shen, ${ }^{\dagger}$ and Ru-Qin Yu \\ State Key Laboratory for Chemo/Biosensing Technology and Chemometrics, College of Chemistry \\ and Chemical Engineering, Hunan University, Changsha, 410082, P. R. China
}

\begin{abstract}
A renewable amperometric immunosensor based on a graphite-paraffin-Schistosoma japonicum antibody ( $\mathrm{SjAb})$ biocomposite electrode has been prepared for the detection of Schistosoma japonicum antigen (SjAg). Competitive ELISA was employed involving HRP-SjAg as a tracer and 3,3',5,5'tetramethylbenzidine (TMB) as a substrate. The product of an enzyme catalytic reaction was detected at $+0.1 \mathrm{~V}$ ( $v s . \mathrm{Ag} / \mathrm{AgCl}$ reference electrode) for measuring the amount of HRP-labeled SjAg binding to the electrode surface. The assay conditions were optimized, including the amount of $\mathrm{SjAb}$ loading in the electrode and HRP-SjAg in the incubation solution, the $\mathrm{pH}$ of the measuring solution and the incubation time. The measuring range was $0.5-30 \mu \mathrm{g} / \mathrm{ml}$ under the optimum conditions. Rabbit serum samples of different infection degree were measured, which demonstrated that the immunosensor meets the demands of clinical analysis. It exhibits some advantages, such as simplicity of fabrication, rapidity of measurement, and satisfactory sensitivity and reproducibility.
\end{abstract}

(Received June 29, 2001; Accepted November 5, 2001)

\section{Introduction}

Schistosomiasis japonicum ( $\mathrm{Sj})$, a parasite disease, still threatens human health in some parts of Asia, Africa and Latin America. About 1.6 billion people in 76 countries have been infected with this parasite. ${ }^{1}$ Thus, the development of fast, robust, selective, easy-to-use, low-cost methods for the determination of $\mathrm{SjAg}$ or $\mathrm{SjAb}$ is of importance for health protection and for further medical treatment. Conventional diagnostic procedures of this disease are often time-consuming, complicated and need expensive instrumentation. ${ }^{2-4}$ These disadvantages have sparked the development of immunosensors.

Electrochemical immunosensors draw interest owing to the relatively simple and inexpensive equipment required. ${ }^{5-8}$ For the design of an immunosensor, antibodies for the target analyte must be immobilized on the transducer surface, and immobilized antibodies should be stable enough to retain the bioactivity during a sufficient lifetime. General methods for the immobilization of $\mathrm{Ab}$ include physical adsorption, covalent attachment and entrapment in a polymer matrix. ${ }^{9-12}$ Another prerequisite for a successful application of an immunosensor is regeneration of the sensing surface after each assay. ${ }^{13}$ Regeneration of the sensing surface is usually accomplished by using solutions of low $\mathrm{pH}$ and high salt concentration. However, such a regeneration process is often incomplete and gives irreproducible results due to strong binding of $\mathrm{Ag}$ with Ab. A possible solution to this problem is to develop singleuse, low-cost immunosensors. ${ }^{14-16}$ Fàbregas et al..$^{17,18}$ have

$\doteqdot$ To whom correspondence should be addressed.

E-mail: Shenguo@cs.hn.cn reported renewable amperometric immunosensors based on graphite powder, methacrylate or epoxy resin and an $\mathrm{Ab}$ composite. The surface of an immunosensor may be renewed through simple polishing.

Carbon paste electrodes (CPE) are also a good choice for the design of immunosensor filling the above two requirements. CPE can easily be modified with an antibody, and the sensing surface of CPE can be regenerated in a rapid and quantitatively reproducible fashion. Encouraged by the success of constructing of a renewable amperometric immunosensor based on a graphite-paraffin-SjAg biocomposite electrode for the detection of $\mathrm{SjAb}$, we tried to use a graphite-paraffin- $\mathrm{SjAb}$ electrode as immunosensor for the determination of $\mathrm{SjAg}$ using a competitive ELISA involving HRP-SjAg as a tracer and $3,3^{\prime}, 5,5^{\prime}$-tetramethylbenzidine (TMB) as a substrate. The determination of $\mathrm{SjAg}$ is necessary for the diagnosis of schistosomiasis, especially during the initial stage.

\section{Experimental}

Apparatus

A 20-ml electrochemical cell equipped with an immunosensor, an $\mathrm{Ag} / \mathrm{AgCl}$ reference electrode and a platinumwire auxiliary electrode was used for the electrochemical measurements. Cyclic voltammetric and chronoamperometric experiments were carried out with a microcomputer-based electrochemical analyzer (Tianjin Lanlike Chemical Electronic Co.) and XJP-821(c) polarographic analyzer (Jiansu Electroanalytical Instruments, Jiansu). All of the electrochemical measurements were performed in a $0.1 \mathrm{~mol} / \mathrm{l}$ citrate phosphate buffer solution. A Model CSS501 thermostat (Chongqing Experimental Equipment, Chongqing, China) was 


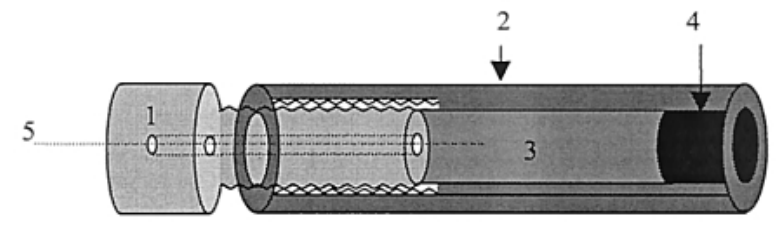

Fig. 1 Configuration of amperometric immunosensor: (1) Teflon nut, (2) Teflon tube, (3) conducting stainless-steel rod, (4) biocomposite, (5) electric wire connected with stainless-steel rod.

used to control the incubation temperature. During amperometric experiments, a magnetic bar was used to stir the solution.

\section{Reagents and buffers}

A $32 \mathrm{kDa}$ molecular antigen of Schistosomiasis japonicum from an adult worm antigen (AWA) was isolated and purified to homogeneity according to a reported method. ${ }^{19} \mathrm{SjAb}$ was prepared through immunizing rabbits with $2500 \mathrm{SjAg}$ for 45 days. The antibody in the infected rabbit serum was purified by precipitation from a saturated ammonium sulfate solution, as described in the literature. ${ }^{20}$ Horseradish peroxidase (HPR) and 3,3',5,5'-tetramethylbenzidine (TMB) were obtained from Sigma. All other chemicals used were of analytical grade. A blocking buffer solution containing $0.1 \% \mathrm{BSA}$ w/w and $1 \mathrm{mM}$ EDTA in $0.1 \mathrm{~mol} / \mathrm{l} \mathrm{Tris-} \mathrm{HCl}(\mathrm{pH} 7.4)$ was used as an incubation solution. The washing solution was a $0.1 \mathrm{~mol} / 1$ Tris-HCl- 0.1 $\mathrm{mol} / \mathrm{l} \mathrm{KCl}$ buffer. A solution of $1 \% \mathrm{BSA}$ in a Tris- $\mathrm{HCl}$ buffer solution was used to dilute the $\mathrm{SjAg}$. All solutions were prepared with doubly distilled water.

Fabrication of a graphite-paraffin-SjAb biocomposite based immunosensor

The configuration of the immunosensor is illustrated in Fig. 1. The immunosensor was prepared as follows: $0.5 \mathrm{~g}$ of graphite powder, $15 \mathrm{mg}$ of $\mathrm{SjAb}$ and $0.5 \mathrm{~g}$ of paraffin were dispersed in a small amount of diethyl ether to obtain a homogeneous carbon paste $(\mathrm{CP})$. The resulting paste was kept at room temperature to evaporate a major part of the ether. The modified CP was packed into a Teflon tube (6 $\mathrm{mm}$ i.d. and $10 \mathrm{~mm}$ deep). A stainless-steel rod was inserted into the tube to pack the carbon paste and achieve electric contact.

\section{Preparation of HRP-SjAg conjugate}

HRP was coupled to $\mathrm{SjAg}$ by the standard periodate method described in the literature. ${ }^{1}$ HRP $(5 \mathrm{mg})$ was dissolved in $0.5 \mathrm{ml}$ of acetate buffer $(0.2 \mathrm{~mol} / \mathrm{l}, \mathrm{pH} 5.6)$, and then oxidized with $0.25 \mathrm{ml}$ of freshly prepared $0.1 \mathrm{~mol} / \mathrm{l} \mathrm{NaIO}_{4}$ for $30 \mathrm{~min}$ in the dark. Glycol $(0.5 \mathrm{ml}, 2.5 \% \mathrm{v} / \mathrm{v})$ was added to the solution and incubated for $30 \mathrm{~min}$ to neutralize any excess periodate. Then, $\mathrm{SjAg}(5 \mathrm{mg})$ was added and the $\mathrm{pH}$ of the solution was adjusted to 9.0 with sodium carbonate buffer. This solution was allowed to stay overnight at $4^{\circ} \mathrm{C}$. A freshly prepared $0.1 \mathrm{ml} \mathrm{NaBH}_{4}(5$ $\mathrm{mg} / \mathrm{ml}$ ) solution was added to reduce the Schiff base formed between HRP and $\mathrm{SjAg}$ for $2 \mathrm{~h}$. The reaction mixture was dialyzed for one day against a $0.1 \mathrm{~mol} / \mathrm{l}$ phosphate buffer, $\mathrm{pH}$ 7.4 , at $4^{\circ} \mathrm{C}$ to remove any non-reacted low-molecular reagents. Further purification was conducted by gel filtration on a Sepharose G-25 column to give the SjAg-HRP conjugate. The conjugate was mixed with glycerd and stored at $-20^{\circ} \mathrm{C}$ until ready to use.

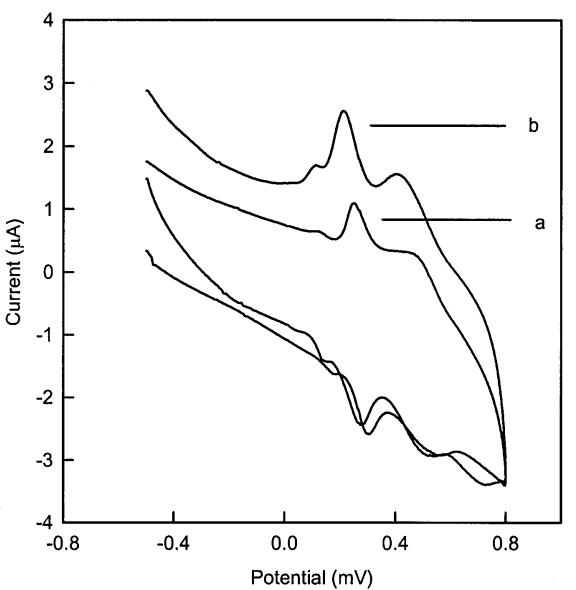

Fig. 2 Cyclic voltammograms of $0.5 \times 10^{-3} \mathrm{~mol} / 1 \mathrm{TMB}$ and $1 \times$ $10^{-3} \mathrm{~mol} / 1 \mathrm{H}_{2} \mathrm{O}_{2}$ in $0.1 \mathrm{~mol} / \mathrm{l} \mathrm{pH} 5$ citrate-phosphate buffer (a) recorded in the absence of $\mathrm{HRP}$, (b) recorded $5 \mathrm{~min}$ after the addition of $1 \mu \mathrm{g} / \mathrm{ml} \mathrm{HRP}$ in solution. Scan rate, $100 \mathrm{mV} / \mathrm{s}$.

\section{Immunoassay procedure}

A competitive enzyme-linked immunoassay was performed on the SjAb-modified electrode surface for the determination of $\mathrm{SjAg}$. The immunosensor was incubated in $1 \mathrm{ml}$ of a blocking buffer solution containing $3 \mu \mathrm{g} / \mathrm{ml} \mathrm{HRP}-\mathrm{SjAg}$ and different concentrations of $\mathrm{SjAg}$ for $30 \mathrm{~min}$ at $25^{\circ} \mathrm{C}$. The free $\mathrm{SjAg}$ competed with the HRP-SjAg for binding to an $\mathrm{SjAb}$-coated electrode surface. The amount of HRP-SjAg binding to the electrode surface was inversely proportional to the amount of the analyte in the sample. The immunosensor was thoroughly rinsed with the washing buffer to remove any unbound tracer. The electrode was placed in an electrochemical cell containing $10 \mathrm{ml}$ of $0.1 \mathrm{~mol} / \mathrm{l}$ citrate-phosphate buffer (pH 5). After adding $100 \mu \mathrm{l}$ of TMB and $\mathrm{H}_{2} \mathrm{O}_{2}$ peroxidase substrate to give a final concentration of $5 \times 10^{-4} \mathrm{~mol} / \mathrm{l}$ and $1 \times 10^{-3} \mathrm{~mol} / \mathrm{l}$, respectively, the reduction current signal due to the mediator was recorded at $+100 \mathrm{mV}$ versus $\mathrm{Ag} / \mathrm{AgCl}$ reference electrode as an assay signal.

\section{Renewal of the immunosensor surface}

Prior to each immunoassay, a small amount of paste was pushed out of the tip, and the surface of immunosensor was polished manually on ultra fine emery-paper until a smooth surface was achieved. The electrode surface was cleaned with doubly distilled water. When not in use, the immunosensor was stored in a refrigerator at $4^{\circ} \mathrm{C}$.

\section{Results and Discussion}

\section{Cyclic voltammetric studies}

Immunoassays are based on the inherent molecularrecognition capabilities of an antibody for the corresponding antigen. Interactions between $\mathrm{Ab}$ and $\mathrm{Ag}$ can be measured indirectly via detection of using labeled molecules, such as a radioisotope, an enzyme or a fluorescent tracer. The enzymelinked label can provide a means to amplify the resulting Ag-Ab complex formation. In the present study, we used HRP-SjAg as a tracer and TMB as an enzyme substrate. We, at first, investigated the effect of HRP on the reaction between TMB and $\mathrm{H}_{2} \mathrm{O}_{2}$. The cyclic voltammograms were taken in a $0.1 \mathrm{~mol} / \mathrm{l}$ citrate-phosphate buffer ( $\mathrm{pH}$ 5) containing $5 \times 10^{-4} \mathrm{~mol} / \mathrm{l} \mathrm{TMB}$ 


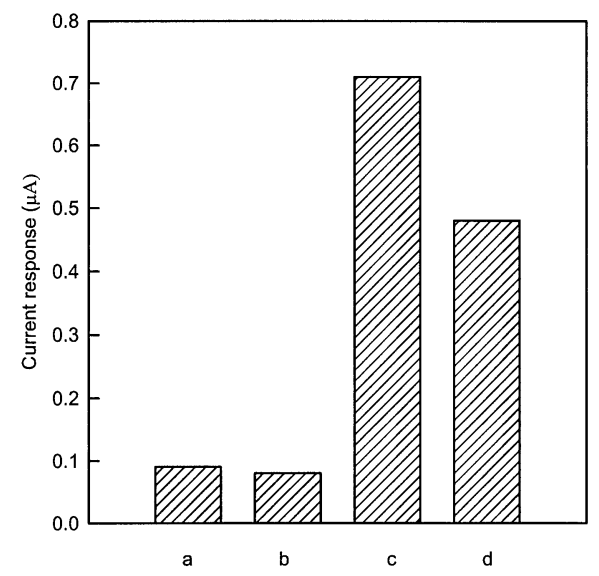

Fig. 3 Amperometric responses of a BSA-modified electrode (a, b) and the $\mathrm{SjAb}$-modified electrode (c, d) in $0.1 \mathrm{~mol} / \mathrm{l}$ citrate-phosphate containing $0.5 \times 10^{-3} \mathrm{~mol} / \mathrm{l} \mathrm{TMB}$ and $1 \times 10^{-3} \mathrm{~mol} / 1 \mathrm{H}_{2} \mathrm{O}_{2}$. The electrodes were incubated with $3 \mu \mathrm{g} / \mathrm{ml} \operatorname{HRP}-\operatorname{SjAg}(\mathrm{a}, \mathrm{c})$ or with 3 $\mu \mathrm{g} / \mathrm{ml}$ HRP-SjAg $+2 \mu \mathrm{g} / \mathrm{ml} \mathrm{SjAg} \mathrm{(b,} \mathrm{d)} \mathrm{for} 40 \mathrm{~min}$.

and $1 \times 10^{-3} \mathrm{~mol} / 1 \mathrm{H}_{2} \mathrm{O}_{2}$ with a scan rate of $100 \mathrm{mV} / \mathrm{s}$ between the potential ranges -500 to $800 \mathrm{mV}$. As shown in Fig. 2(a), a two-electron redox behavior was observed for TMB. Figure 2(b) is the cyclic voltammogram recorded $5 \mathrm{~min}$ after the addition of $1 \mu \mathrm{g} / \mathrm{ml} \mathrm{HRP}$. The presence of HRP results in an increase of the cathodic current. This demonstrates that TMB and $\mathrm{H}_{2} \mathrm{O}_{2}$ are favorable for a sensitive amperometric detection of HRP. The graphite-paraffin electrode offers effective detection of the biocatalytic product.

\section{Immunosensor response to SjAg}

To investigate the amperometric response of the immunosensor to the analyte, an SjAb-modified paraffingraphite composite electrode and a BSA-modified paraffingraphite composite electrode were incubated with $3 \mu \mathrm{g} / \mathrm{ml} \mathrm{HRP-}$ $\mathrm{SjAg}$ in the absence and presence of $2 \mu \mathrm{g} / \mathrm{ml} \mathrm{SjAg}$ for $40 \mathrm{~min}$. As shown in Fig. 3, the BSA-modified paraffin-graphite composite electrode shows a small amperometric signal in the absence (a) or presence (b) of the analyte only due to nonspecific binding. In contrast to the BSA-modified paraffingraphite composite electrode, the $\mathrm{SjAb}$-modified paraffingraphite composite electrode has a large amperometric response to HRP-SjAg, as shown in Fig. 3(c), which indicates that HRP$\mathrm{SjAg}$ binds with $\mathrm{SjAb}$ immobilized on the electrode surface through specific immunoreaction. The presence of free $\mathrm{SjAg}$ in the incubation solution (d) decreases the amperometric response owing to decreasing the amount of HRP-SjAg by competitive binding of $\mathrm{SjAg}$ to the immunosensor surface. The decrease in the current was about $40 \%$ of the current due to HRP-SjAgspecific binding to $\mathrm{SjAb}$ immobilized on the electrode surface (response current substrates base-line current). This demonstrates that the proposed immunosensor exhibits a welldefined response to the analyte. In order to promote an analytical performance of the immunosensor, the effects of several experimental parameters were investigated.

\section{Effect of SjAb loading in an immunosensor}

Because the assay performance involves $\mathrm{SjAg}$ binding with $\mathrm{SjAb}$ immobilized on an electrode surface, it is necessary to define the amount of $\mathrm{SjAb}$ incorporated in a carbon-paraffin composite electrode, which influences the amount of tracer that binds to the electrode surface in the incubation step and the

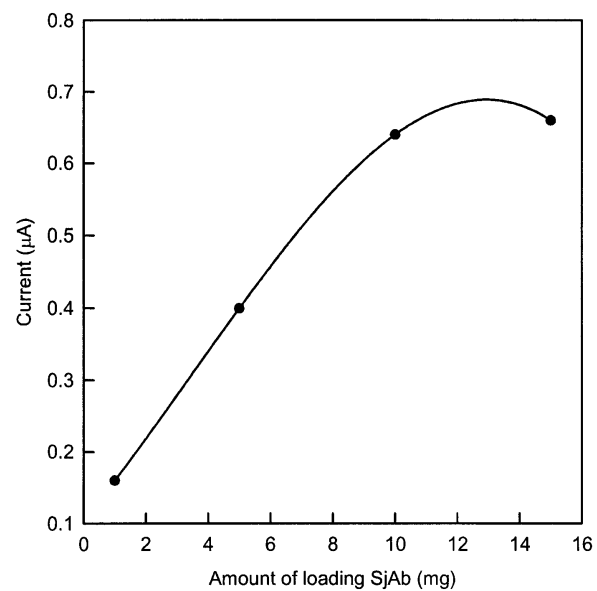

Fig. 4 Effect of $\mathrm{SjAb}$ loading in a carbon-paraffin composite electrode on the amperometric response of the immunosensor. Immunosensor was incubated with $3 \mu \mathrm{g} / \mathrm{ml} \mathrm{HRP-SjAg}$ for $40 \mathrm{~min}$.

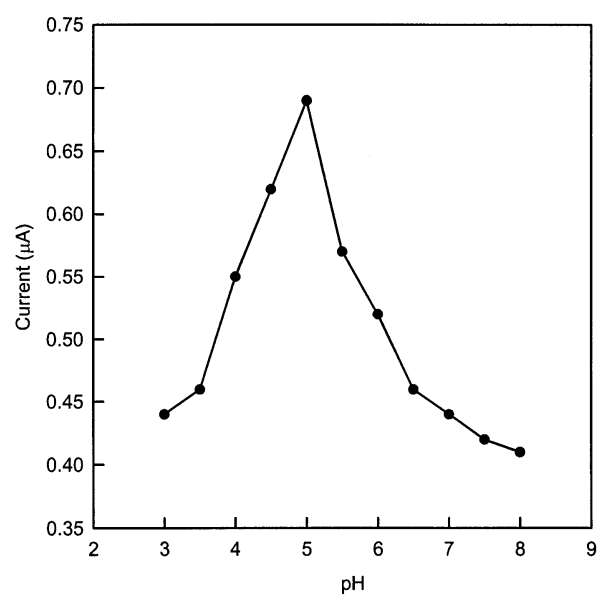

Fig. 5 Effect of the $\mathrm{pH}$ of a citrate-phosphate buffer solution on the amperometric response of an immunosensor. The immunosensor was incubated with $3 \mu \mathrm{g} / \mathrm{ml} \mathrm{HRP-SjAg}$ for $40 \mathrm{~min}$.

amperometric response signal. Immunosensors modified with different amounts of $\mathrm{SjAb}$ were incubated in $3 \mu \mathrm{g} / \mathrm{ml} \mathrm{HRP-SjAg}$ for $40 \mathrm{~min}$, and the amperometric responses were measured. The results (Fig. 4) show that the amperometric response increases with raising the $\mathrm{SjAb}$ loading in the carbon-paraffin composite electrode. To maximize the signal response and to minimize the used amount of $\mathrm{SjAb}$, a $15 \mathrm{mg}$ loading of $\mathrm{SjAb}$ in a carbon-paraffin composite was selected to prepare the immunosensor for subsequent experiments.

\section{Effect of the $\mathrm{pH}$}

The $\mathrm{pH}$ of the measuring solution influences the activity of the enzyme and catalytic product. In order to optimize the $\mathrm{pH}$ of the measuring solution for an immunoassay, the immunosensor was incubated in $3 \mu \mathrm{g} / \mathrm{ml} \mathrm{HRP}-\mathrm{SjAg}$ for $40 \mathrm{~min}$, and the amperometric response was measured in a $0.1 \mathrm{~mol} / \mathrm{l}$ citrate-phosphate buffer of different $\mathrm{pH}$. Figure 5 shows the effect of the $\mathrm{pH}$ on the magnitude of the amperometric signal. Because the current response arrives at a maximum value at $\mathrm{pH}$ 5.0 , we used a $\mathrm{pH}$ of 5.0 of a citrate-phosphate buffer for the amperometric measurements. 


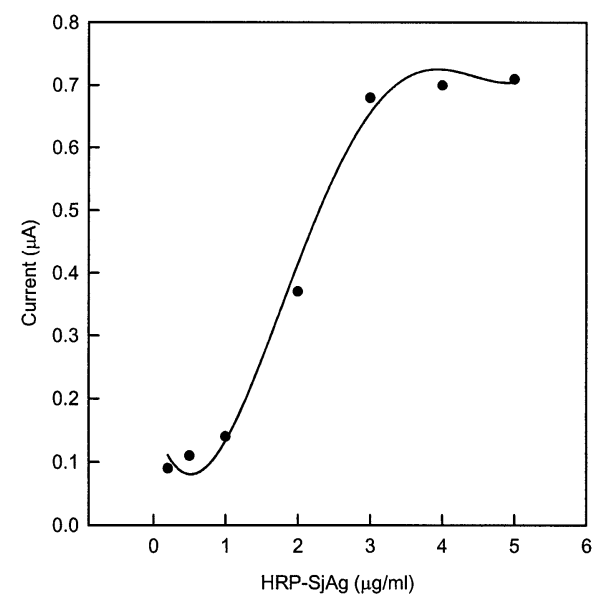

Fig. 6 Effect of the concentration of HRP-SjAg in the incubation solution on the amperometric response of the immunosensor. The immunosensor was incubated with different concentrations of HRP$\mathrm{SjAg}$ for $30 \mathrm{~min}$.

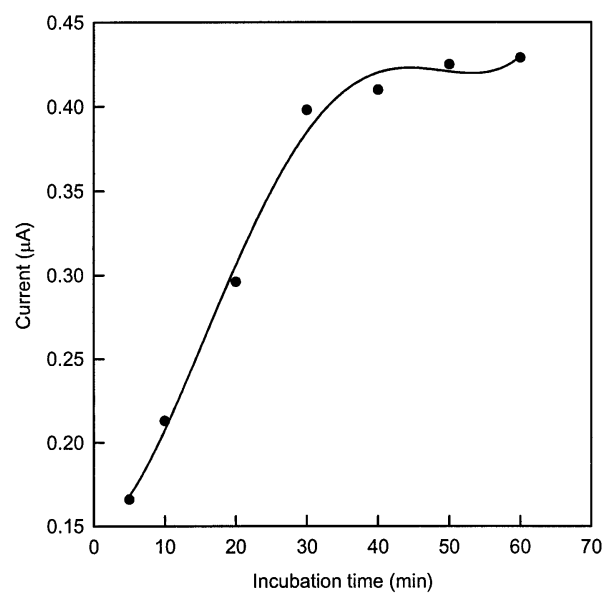

Fig. 7 Effect of the incubation time on the amperometric response of immunosensor. The immunosensor was incubated with $3 \mu \mathrm{g} / \mathrm{ml}$ HRP-SjAg $+5 \mu \mathrm{g} / \mathrm{ml} \mathrm{SjAg}$ for different time.

\section{Effect of the HRP-SjAg concentration}

The concentration of the HRP-SjAg used in a competitive immunoassay also influences the amperometric response of the immunosensor. To optimize the HRP-SjAg concentration, the immunosensor was incubated with increasing concentrations of HRP-SjAg for $30 \mathrm{~min}$. As shown in Fig. 6, the amperometric response increases with an increase of the concentration of HRP-SjAg up to $3 \mu \mathrm{g} / \mathrm{ml}$, and then trends to saturate. Thus, 3 $\mu \mathrm{g} / \mathrm{ml} \mathrm{HRP-SjAg} \mathrm{in} \mathrm{an} \mathrm{incubation} \mathrm{solution} \mathrm{was} \mathrm{selected} \mathrm{to}$ maximize the response and to minimize the amount of HRPSjAg used.

\section{Effect of the incubation time}

The effect of the incubation time in which the tracer and the analyte were allowed to competitively bind to the immobilized $\mathrm{SjAb}$ was studied. The immunosensor was incubated with a solution containing $3 \mu \mathrm{g} / \mathrm{ml} \mathrm{HRP-SjAg}$ and $5 \mu \mathrm{g} / \mathrm{ml} \mathrm{SjAg}$ for different times. The results are depicted in Fig. 7, which show that the amperometric signal increased rapidly with the incubation time up to $30 \mathrm{~min}$, and then trended to saturate. Thus $30 \mathrm{~min}$ was selected as the optimal incubation time to

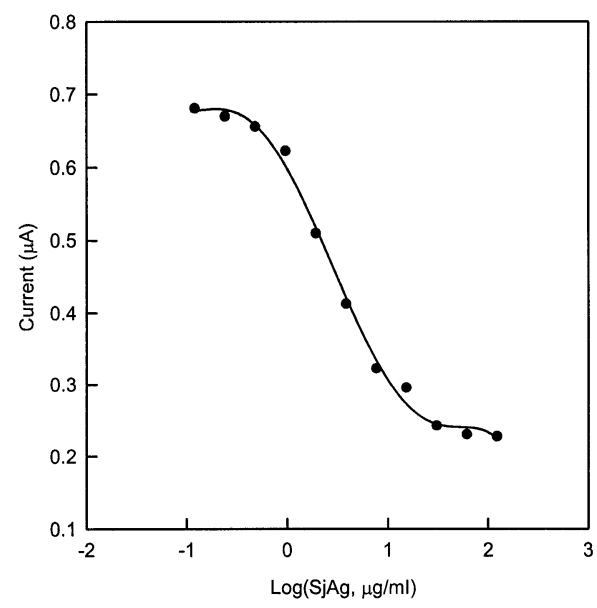

Fig. 8 Calibration graph showing current responses versus the concentration of $\mathrm{SjAg}$. The immunosensor was incubated with 3 $\mu \mathrm{g} / \mathrm{ml}$ HRP-SjAg solutions containing different concentrations of $\mathrm{SjAg}$ for $30 \mathrm{~min}$.

maximize the signal and to minimize the assay time.

Determination of SjAg with the immunosensor

For the determination of $\mathrm{SjAg}$, a competitive immunoassay was performed under optimized conditions. Figure 8 displays the competitive curve. As expected for competitive assays according to the four-parameters-logistic equation of Rodbard, ${ }^{21}$

$$
y=d+(a-d) /\left(1+(x / c)^{b}\right),
$$

where $a$ represents the upper asymptote of the curve, $b$ the slope of the curve at the midpoint, $c$ the analyte concentration at the midpoint, $d$ the residual current, $x$ the analyte concentration and $y$ the test signal. A sigmoidal relationship exists between the assay response and the free analyte concentration. The measuring range was $0.5-30 \mu \mathrm{g} / \mathrm{ml}$ and the relative standard deviation was below $6.8 \%(n=4)$. This demonstrates that the immunosensor offered a reproducible sensing surface by polishing after each measurement. The applicability of the immunosensor for the detection of the $\mathrm{SjAg}$ in rabbit serum has been demonstrated. Four rabbit serum samples with different infection degrees were assayed. The results are given in Table 1, which demonstrate that the immunosensor meets the requirements for clinical analysis for evaluating the infection degree by $\mathrm{SjAg}$ in serum and the diagnosis of schistosomiasis.

\section{Conclusions}

The use of an SjAb-modified paraffin-graphite composite electrode for the determination of $\mathrm{SjAg}$ in serum samples was examined in this paper. Competitive ELISA formats were adopted with HRP-SjAg as a tracer. The developed system permits a reliable determination of $\mathrm{SjAg}$ in serum. It also offers some advantages, such as simplicity of fabrication, rapidity of measurement and being renewable by simple manipulation.

\section{Acknowledgements}

This work was supported by the National Natural Science Foundation of China (Grants No. 29975006, 20075006), the 
Table 1 Determination of $\mathrm{SjAg}$ in a rabbit serum sample

\begin{tabular}{|c|c|c|c|}
\hline $\begin{array}{l}\text { Rabbit serum } \\
\text { sample }\end{array}$ & Infection degree ${ }^{a}$ & Current/ $\mu \mathrm{A}$ & 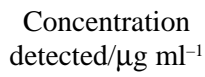 \\
\hline $1^{\#}$ & 1000 & 0.60 & 1.1 \\
\hline $2^{\#}$ & 2000 & 0.56 & 1.4 \\
\hline $3^{\#}$ & 3500 & 0.51 & 1.9 \\
\hline $4^{\#}$ & 5000 & 0.44 & 3.2 \\
\hline
\end{tabular}

a. The infection degree is designed to the number of Schistosoma japonicum in immunizing rabbits experiments.

Foundation for Ph D Thesis Research (No. 20010532008) and Foundation of Scientific Commission of Hunan Province.

\section{References}

1. Y. H. Liu, X. X. Liu, C. C. Song, and X. H. Yu, "Immunology and Immunodiagnosis Parasitic Diseases", 1991, Jiangsu Science-Technology Press.

2. X. Bossuyt, A. Bogaerts, G. Schiettekatte, and N. Blankaert, Clin. Chem., 1998, 44, 760.

3. R. Hawkes, Anal. Biochem., 1982, 119, 142.

4. M. Maeda and A. Tsuji, Anal. Chim. Acta, 1985, 167, 241.

5. J. Wang, Anal. Chem., 1999, 71, 328R.

6. E. Crowley, C. O'sullivan, and G. G. Guilbault, Anal Chim. Acta, 1999, 389, 171
7. R. M. Pemberton, J. P. Hart, and J. A. Foulkes, Electrochim. Acta, 1998, 43, 3567.

8. I. Abdel-Hamid, P. Atanasov, A. L. Ghindilis, and E. Wilkins, Sens. Actuators B, 1998, 49, 202.

9. C. Padeste, A. Grubelnik, and L. Tiefenauer, Anal. Chim Acta, 1998, 374, 167.

10. J. Wang, B. Tian, and K. R. Rogers, Anal. Chem., 1998, 70, 1682.

11. A. Sargent and O. A. Sadik, Anal. Chim. Acta, 1998, 376, 125.

12. D. Barnett, D. G. Laing, S. Skopec, O. Sadik, and G. G. Wallace, Anal. Lett., 1994, 27, 2417.

13. C. K. O'Sullivan, R. Vaughan, and G. G. Guilbault, Anal. Lett., 1999, 32, 2353.

14. J. Wang, P. V. A. Pamidi, and K. R. Rogers, Anal. Chem., 1998, 70, 1171

15. K. S. Lee, T. H. Kim, M. C. Shin, W. Y. Lee, and J. K. Park, Anal. Chim. Acta, 1999, 380, 17.

16. M. A. González-Martínez, R. Puchades, A. Maquieira, I. Ferrer, M. P. Marco, and D. Barceló, Anal. Chim. Acta, 1999, 386, 201.

17. M. Santandreu, F. Céspedes, S. Alegret, and E. Fàbregas, Anal. Chem., 1997, 69, 2080.

18. M. Santandreu, S. Alegret, and E. Fàbregas, Anal. Chim. Acta, 1999, 396, 181.

19. S. P. Wang, X. F. Zeng, and X. Y. Yi, Chin. J. Parasitol. Parasitic Dis., 1995, 13, 25.

20. S. P. Wang, X. F. Zeng, and X. Y. Yi, Chin. J. Zoonoses, 1992, 4,14

21. D. Rodbard, Clin. Chem., 1974, 20, 1255. 